\title{
Article \\ Intuitionistic Fuzzy (IF) Overlap Functions and IF-Rough Sets with Applications
}

\author{
Xiaofeng Wen ${ }^{1}$, Xiaohong Zhang ${ }^{2, *}$ and Tao Lei ${ }^{2,3}$ \\ 1 School of Mathematics \& Data Science, Shaanxi University of Science and Technology, \\ Xi'an Weiyang University Park, Xi'an 710021, China; 1909015@sust.edu.cn \\ 2 Shaanxi Joint Laboratory of Aritificial Intelligence, Shaanxi University of Science and Technology, \\ Xi'an 710021, China; leitao@sust.edu.cn \\ 3 School of Electronic Information and Artificial Intelligence, Shaanxi University of Science and Technology, \\ Xi'an 710021, China \\ * Correspondence: zhangxiaohong@sust.edu.cn
}

check for

updates

Citation: Wen, X.; Zhang, X.; Lei, T. Intuitionistic Fuzzy (IF) Overlap Functions and IF-Rough Sets with Applications. Symmetry 2021, 13, 1494 https://doi.org/10.3390/sym13081494

Academic Editor: Saeid Jafari

Received: 8 July 2021

Accepted: 12 August 2021

Published: 14 August 2021

Publisher's Note: MDPI stays neutral with regard to jurisdictional claims in published maps and institutional affiliations.

Copyright: (c) 2021 by the authors. Licensee MDPI, Basel, Switzerland. This article is an open access article distributed under the terms and conditions of the Creative Commons Attribution (CC BY) license (https:// creativecommons.org/licenses/by/ $4.0 /)$

\begin{abstract}
Overlap function (which has symmetry and continuity) is widely used in image processing, data classification, and multi-attribute decision making problems. In recent years, theoretical research on overlap function has been extended to interval valued overlap function and lattice valued overlap function, but intuitionistic fuzzy overlap function (IF-overlap function) has not been studied. In this paper, the concept of IF-overlap function is proposed for the first time, then the generating method of IF-overlap function is given. The representable IF-overlap function is defined, and the concrete examples of representable and unrepresentable IF-overlap functions are given. Moreover, a new class of intuitionistic fuzzy rough set (IF-roght set) model is proposed by using IF-overlap function and its residual implication, which extends the IF-rough set model based on intuitionistic fuzzy triangular norm, and the basic properties of the new intuitionistic fuzzy upper and lower approximate operators are analyzed and studied. At the same time, the established IF-rough set based on IF-overlap function is applied to MCDM (multi-criteria decision-making) problems, the intuitionistic fuzzy TOPSIS method is improved. Through the comparative analysis of some cases, the new method is proved to be flexible and effective.
\end{abstract}

Keywords: intuitionistic fuzzy sets; intuitionistic fuzzy overlap function; intuitionistic fuzzy triangular norm; IF-TOPSIS methods; MCDM

\section{Introduction}

Fuzzy set theory is a very effective mathematical tool to analyze and deal with inaccurate and incomplete information [1]. It plays an increasingly important role in many practical engineering fields, including fuzzy preference relationship [2], fuzzy information clustering [3], fuzzy granularity calculation [4], attribute decision problem [5], etc. According to practical problems, different forms of fuzzy sets are proposed, such as intervalvalued fuzzy sets [6], intuitionistic fuzzy sets [7-9], etc. Rough set theory [10] proposed by Pawlak is also a mathematical tool to deal with fuzzy and uncertain knowledge, and has been successfully applied to machine learning, decision analysis, process control, pattern recognition, data mining and other fields. Dubois and Prade combined the two theories and proposed the concept of fuzzy rough set for the first time [11]. Since then, studies on fuzzy rough sets have become more abundant and indepth [12-16]. Because fuzzy rough sets are a special case of intuitionistic fuzzy rough sets (IF-rough sets), this paper focuses on intuitionistic fuzzy rough sets.

IF set is an expansion and development that has great influence on fuzzy set theory $[7,9,17]$. It has been successfully applied to the fields of decision analysis and pattern recognition $[18,19]$ and intuitionistic fuzzy decision analysis [20,21]. Compared with the fuzzy set, which only indicates the degree to which an element belongs to a set, 
the IF set describes the membership degree and non-membership degree of an element to a set, and more accurately expresses the relationship between them, which adds a new field of vision for the solution of multi-attribute decision making problems. Subsequently, many concepts of IF have been proposed, such as IF relation [22], IF triangular norm [23], IF implication operator [24], etc. When dealing with practical problems, IF set and rough set theory are combined to solve such problems because of the roughness of information. In 2003, De Cock et al. presented some basic and important properties and conclusions of IF-rough set [25]. As an important continuous operator, IF triangular norm plays an important role in IF-rough sets. De Cock et al. defined a pair of upper and lower IF-rough approximation operators based on IF implication operators and IF triangular norm, studied the properties of IF-rough operators, and further extended the IF-rough set model [26]. On the basis of systematic generalization of existing work, Zhang et al. extended one domain to two domains [27] to study IF-rough sets.

Since triangular norm is widely used in solving practical problems, the study of their extensive forms in application is also of great importance. The overlap function is the extension of continuous triangular norm and is widely used in image processing, data classification and multi-attribute decision making. Overlap functions as unassociative connectives in fuzzy logic has been studied by many scholars. Overlap function [28] was proposed by Bustince et al. in 2009. This concept is taken from some practical problems related to image processing and classification. In image problem processing, Bustince scholars use binary operators called constrained equivalent functions to calculate the threshold of an image [29]. In the classification problem, Amo scholars use overlaps to discuss the evaluation of resulting classification when the research objects is unclear classification system [30]. In recent years, some generalizations of overlap function have been proposed, such as n-dimensional overlap function and general function [31], interval valued overlap function [32-34], etc., which have promoted theoretical research and practical application of overlap function. However, the existing definitions have limitations in solving practical problems with intuitionistic fuzzy information. In view of this limitation, this paper puts forward the definition of IF-overlap function, and studies some of its properties and representations.

Because the overlap function has a wide range of applications, IF-overlap function and IF-rough set can be studied together, and a broader IF-rough set model can be proposed to expand the application range of IF-rough set in practical problems. IF-overlap function, as a non-associative binary function, can be widely used in decision making problems based on fuzzy preference relation, which can overcome the defect of associative property of continuous IF triangular norm in practical problems, and has a better effect in dealing with uncertain multi-attribute decision making problems. Therefore, on the basis of IF triangular norm, a broader IF-rough sets is proposed, that is, IF-rough set based on IFoverlap function.

There are two main reasons for this study: One is the rough set theory is an important tool to deal with uncertain information, however, the classical rough set is restricted because of its strict conditions, in order to expand the application scope of rough set theory, we found that IF the introduction of the theory makes a lot of problems to solve, through different logical operator combining the IF theory and rough set theory, such as IF-overlap function, enriched the theory of rough set. Secondly, for the application of MCDM problem, after studying and comparing many existing methods, we found that the existing methods still have some limitations, for example, the continuity of triangular norm operator may be invalid in the complex IF environment. This paper analyzes the limitations of these methods in theory and application, and puts forward a new method to solve the MCDM problem. Experiments show that our method is more suitable for practical needs and more flexible when dealing with problems. In addition, through changing attribute values of $\alpha$, this method can get all the results obtained by existing methods.

The rest of the paper is structure as following: we list some preliminary concepts and results in Section 2. Next we give the definition of the IF-overlap function, given 
the general generation method of the IF-overlap function and some examples to account for explain, moreover we give the definition of the representable and unrepresentable IFoverlap function and concrete example in Section 3. In Section 4, we establish the IF-rough set model based on IF-overlap function, and discuss the basic properties of this model and give some examples. In Section 5, we put forward the MCMD problem method of the IF-rough set model based on IF-overlap function, the steps and calculation formula of this method are listed. Then we propose the concrete example, and comparison analysis among our method and other methods. Finally, we conclude our work with a summary of the paper in Section 6, and also outline future research.

\section{Preliminary Concepts and Results}

In this section we recall several fundamental conceptions relates to overlap function, intuitionistic fuzzy sets and rough sets.

\subsection{Overlap Function}

Definition 1 ([28]). A bivariate function $O:[0,1]^{2} \rightarrow[0,1]$ is called an overlap function, if for every $a, b, c \in[0,1]$, the following conditions holds:
(O1) $O(a, b)=O(b, a)$;
(O2) $O(a, b)=0 \Leftrightarrow a b=0$;
(O3) $O(a, b)=1 \Leftrightarrow a b=1$;
(O4) $O(a, b) \leq O(a, c)$ if $b \leq c$;
$(\mathrm{O} 5) \mathrm{O}$ is continuous.

Definition 2 ([28]). Let $O:[0,1]^{2} \rightarrow[0,1]$ be an overlap function, then for every $a, b \in[0,1]$, the bivariate function $R_{O}:[0,1]^{2} \rightarrow[0,1]$ defined by,

$$
R_{O}(a, b)=\max \{c \in[0,1] \mid O(a, c) \leq b\}
$$

then $R_{O}$ is the residual implication induced from overlap function $O$.

Definition 3 ([32]). An interval-valued overlap function is a mapping $\widehat{O}: L^{I} \times L^{I} \rightarrow L^{I}$ that respects the following conditions ( where $L^{I}=\left\{\left[x_{1}, x_{2}\right] \mid\left[x_{1}, x_{2}\right] \subseteq[0,1], x_{1} \leq x_{2}\right\}$ ):

(Ô1) $\widehat{O}$ is commutative;

$(\widehat{O} 2) \widehat{O}=[0,0] \Leftrightarrow X=[0,0] \vee Y=[0,0]$;

( $\widehat{O} 3) \widehat{O}=[1,1] \Leftrightarrow X=Y=[1,1]$;

(Ô) $\widehat{O}(Y, X) \leq \widehat{O}(Z, X)$ if $Y \leq Z$;

(Ô5) $\widehat{O}$ is Moore-continuous.

\subsection{Fuzzy Sets Theory}

Definition 4 ([1]). The fuzzy set(or fuzzy subset) on argument domain $X$ is A mapping from $X$ to $[0,1]$ (called membership function) :

$$
\mu_{A}: X \rightarrow[0,1]
$$

for every $x \in X, \mu_{A}(x)$ called the membership of $x$ with respect to $A$.

Definition 5 ([7]). The IF set on the argument $X$ is A defined as follows:

$$
A=\left\{\left\langle x, \mu_{A}(x), v_{A}(x)\right\rangle \mid x \in X\right\}
$$

where, $\mu_{A}(x): X \rightarrow[0,1]$ and $v_{A}(x): X \rightarrow[0,1]$ respectively represent the membership and non-membership of $x$ belong to $A$, and satisfy $0 \leq \mu_{A}(x)+v_{A}(x) \leq 1$.

As the same time, general fuzzy set is denote by : $A=\left\{\left\langle x, \mu_{A}(x), 1-\mu_{A}(x)\right\rangle \mid x \in X\right\}$, obviously. At this point, the IF set degenerates into a general fuzzy set. 
Definition 6 ([7]). Let $A$ and $B$ be intuitionistic fuzzy sets, where

$$
A=\left\{\left\langle x, \mu_{A}(x), v_{A}(x)\right\rangle \mid x \in X\right\}, B=\left\{\left\langle x, \mu_{B}(x), v_{B}(x)\right\rangle \mid x \in X\right\},
$$

then the order and operation are defined as follows:

(1) $A \subseteq B \Leftrightarrow \mu_{A}(x) \leq \mu_{B}(x)$ and $_{A}(x) \geq v_{B}(x)$, for each $x \in X$;

(2) $A \cap B=\left\{\left\langle x, \min \mu_{A}(x), \mu_{B}(x), \max v_{A}(x), v_{B}(x)\right| x \in X\right\}$;

(3) $A \cup B=\left\{\left\langle x, \max \mu_{A}(x), \mu_{B}(x), \min v_{A}(x), v_{B}(x)\right| x \in X\right\}$;

(4) $A^{c}=\left\{\left\langle x, v_{A}(x), \mu_{A}(x) \mid x \in X\right\rangle\right\}$;

(5) $\lambda A=\left\{\left\langle x, 1-\left(1-\mu_{A}(x)\right)^{\lambda}, v_{A}(x)^{\lambda}\right\rangle\right\}$.

IF sets assign to each element $x$ of the universe both a degree of membership $\mu_{A}(x)$ and one of non-membership $v_{A}(x)$ such that $0 \leq \mu_{A}(x)+v_{A}(x) \leq 1$. This hesitation is quantified for each $x$ in $X$ by the number $\pi_{A}(x)=1-\mu_{A}(x)-v_{A}(x)$.

It is well-known IF sets are equivalent of L-fuzyy sets [8]. Let $\left(L^{*}, \leq_{L^{*}}\right)$ be the complete bounded lattice defined by:

$$
\begin{gathered}
L^{*}=\left\{\left\langle x_{1}, x_{2}\right\rangle \mid x_{1}+x_{2} \leq 1\right\} \\
\left(x_{1}, x_{2}\right) \leq_{L^{*}}\left(y_{1}, y_{2}\right) \Leftrightarrow x_{1} \leq y_{1}, x_{2} \geq y_{2} .
\end{gathered}
$$

The units of this lattice are denoted $0_{L^{*}}=\langle 01\rangle, 1_{L^{*}}=\langle 1,0\rangle$. For each element $x \in X$, by $x_{1}$ and $x_{2}$ we denote its first and second components, respectively. An IF set $A$ in a universe $X$ is a mapping from $X$ to $L^{*}$. For every $x \in X$, the value $\mu_{A}(x)=(A(x))_{1}$ is called the membership degree of $x$ to $A$; the value $v_{A}(x)=(A(x))_{2}$ is called the nonmembership degree of $x$ to $A$; and the value $\pi_{A}(x)=1-\mu_{A}(x)-v_{A}(x)$ is called the hesitation degree of $x$ to $A$.

Definition 7 ([22]). An IF relation $R$ on $U$ is an IF set of $U \times U$, i.e., $R$ is given be

$$
\left.R=\left\{\langle x, y\rangle, \mu_{R}(x, y), v_{R}(x, y)\right\rangle \mid(x, y) \in U \times U\right\},
$$

where $\mu_{R}: U \times U \rightarrow[0,1]$ and $v_{R}: U \times U \rightarrow[0,1]$ satisfy $0 \leq \mu_{R}(x, y)+v_{R}(x, y) \leq 1$ for all $(x, y) \in U \times U$.

Let $R$ be an IF relation on $U, R$ is called reflexive if $\mu_{R}(x, y)=1$ and $v_{R}(x, y)=0$ for all $x \in U ; R$ is called symmetric if $\mu_{R}(x, y)=\mu_{R}(y, x)$ and $v_{R}(x, y)=v_{R}(y, x)$ for all $(x, y) \in U \times U ; R$ is called transive if for all $(x, z) \in U \times U$,

$$
\mu_{R}(x, z) \geq \vee_{y \in U}\left[\mu_{R}(x, y) \wedge \mu_{R}(y, z)\right]
$$

and

$$
v_{R}(x, z) \leq \wedge_{y \in U}\left[v_{R}(x, y) \vee v_{R}(y, z)\right]
$$

Definition 8 ([23]). An IF triangular norm is a mapping $T: L^{*} \times L^{*} \rightarrow L^{*}$ which satisfy the following conditions:

(1) $T\left(1_{L^{*}}, x\right)=x$, for any $x \in L^{*}$;

(2) $T(x, y)=T(y, x)$, for any $x, y \in L^{*}$;

(3) If $x \leq_{L^{*}} u, y \leq_{L^{*}} v$, then $T(x, y) \leq_{L^{*}} T(u, v)$, for any $x, y, u, v \in L^{*}$;

(4) $T(x, T(y, z))=T(T(x, y), z))$, for any $x, y, z \in L^{*}$.

\subsection{Fuzzy Rough Sets Theory}

Definition 9 ([10]). Let $(U, R)$ be a Pawlak space, that $R$ is an equivalence relation on the argument domain $U$, if $A$ is a fuzzy set, then a pair of lower and upper approximations on $(U, R)$ is defined as follows:

$$
\begin{aligned}
& \underline{A}_{R}(x)=\min \left\{A(y) \mid y \in[x]_{R}, x \in U\right\} \\
& \bar{A}_{R}(x)=\max \left\{A(y) \mid y \in[x]_{R}, x \in U\right\} .
\end{aligned}
$$


where, $[x]_{R}$ represents the equivalence class about $x$, and called $\underline{A}_{R}$ and $\bar{A}_{R}$ are the lower and upper approximations of the fuzzy set $A$ with respect to $(U, R)$.

If $\underline{A}_{R}=\bar{A}_{R}$, then $A$ is a defined fuzzy set. On the contrary, $A$ is called fuzzy rough set.

Definition 10 ([24]). Let $U$ be a nonempty and finite universe of discourse and $R \in I F(U \times U)$, the pair $(U, R)$ is called an IF approximation space. For any $A \in I F(U)$, the lower and upper approximation of $A$ w.r.t $(U, R)$, denoted by $\underline{R}_{A}$ and $\bar{R}_{A}$, are two intuitionistic fuzzy sets and defined as follows:

$$
\begin{aligned}
& \underline{R}_{A}=\left\{\left\langle x, \mu_{\underline{R}_{A}}(x), v_{\underline{R}_{A}}(x)\right\rangle \mid x \in U\right\} ; \\
& \bar{R}_{A}=\left\{\left\langle x, \mu_{\bar{R}_{A}}(x), v_{\bar{R}_{A}}(x)\right\rangle \mid x \in U\right\} .
\end{aligned}
$$

where

$$
\begin{aligned}
& \mu_{\underline{R}_{A}}(x)=\wedge_{y \in U}\left[v_{R}(x, y) \vee \mu_{A}(y)\right] ; \\
& v_{\underline{R}_{A}}(x)=\vee_{y \in U}\left[\mu_{R}(x, y) \wedge v_{A}(y)\right] . \\
& \mu_{\bar{R}_{A}}(x)=\vee_{y \in U}\left[\mu_{R}(x, y) \wedge \mu_{A}(y)\right] ; \\
& v_{\bar{R}_{A}}(x)=\wedge_{y \in U}\left[v_{R}(x, y) \vee v_{A}(y)\right] .
\end{aligned}
$$

The pair $\left(\underline{R}_{A}, \bar{R}_{A}\right)$ is called the IF-rough set of $A$ w.r.t. $(U, R), \underline{R}_{A}$ and $\bar{R}_{A}$ are referred to as a lower and upper IF-rough approximation operators, respectively.

Definition 11 ([26]). Let $T$ be an IF triangular norm, I be an IF implicator, and $R$ be an IF equivalence relation on $U$. Together they constitute the approximation space $(U, R, T, I)$. For any IF set $A$ in $U$, the lower and upper approximation of $A$ are the IF sets $R \downarrow_{I} A$ and $R \uparrow_{T} A$ in $U$ defined by:

$$
\begin{aligned}
& R \downarrow_{I} A(y)=\inf _{x \in U} I(R(x, y), A(x)), \\
& R \uparrow_{T} A(y)=\sup _{x \in U} I(R(x, y), A(x)) .
\end{aligned}
$$

for all $y \in U$.

$A$ is called definable if and only if $R \downarrow_{I} A(y)=R \uparrow_{T} A(y)$. Conversely, the couple $\left(R \downarrow_{I} A, R \uparrow_{T} A\right)$ is an IF-rough set.

\section{Intuitionistic Fuzzy Overlap Function}

This section first gives the definition of the intuitionistic fuzzy overlap function (IFoverlap function), then gives the general generation method of the IF-overlap function, and then gives the definition of the representable IF-overlap function and gives the concrete example.

Definition 12. An IF-overlap function is a mapping $\widetilde{O}: L^{*} \times L^{*} \rightarrow L^{*}$ that respects the following conditions, for any $x, y, z \in L^{*}$,

( $\widetilde{O} 1)$ Commutativity: $\widetilde{O}(x, y)=\widetilde{O}(y, x)$;

( $\widetilde{O} 2)$ Boundary condition: $\widetilde{O}(x, y)=0_{L^{*}}$ if and only if $x=0_{L^{*}}$ or $y=0_{L^{*}}$;

( $\widetilde{O} 3)$ Boundary condition: $\widetilde{O}(x, y)=1_{L^{*}}$ if and only if $x=y=1_{L^{*}}$;

(ত̈4) Monotonicity: $\widetilde{O}(x, y) \leq_{L^{*}} \widetilde{O}(x, z)$ if $y \leq_{L^{*}} z$;

( $\widetilde{O} 5)$ Continuity: $\widetilde{O}$ is continuous, i.e., $\forall i \in I, y_{i} \in L^{*}, \widetilde{O}\left(x, \vee_{i \in I} y_{i}\right)=\vee_{i \in I} \widetilde{O}\left(x, y_{i}\right)$ and $\widetilde{O}\left(x, \wedge_{i \in I} y_{i}\right)=\wedge_{i \in I} \widetilde{O}\left(x, y_{i}\right)$.

Proposition 1. Let $O$ be an overlap function. Define the function $\widetilde{O}$ as follows: for every $x=$ $\left(x_{1}, x_{2}\right), y=\left(y_{1}, y_{2}\right) \in L^{*}$,

$$
\widetilde{O}(x, y)=\left\langle O\left(x_{1}, y_{1}\right), 1-O\left(1-x_{2}, 1-y_{2}\right)\right\rangle
$$


then $\widetilde{O}$ is an IF-overlap function.

Proof. Prove $\widetilde{O}$ satisfied the conditions in Definition 12 as follows:

$(\widetilde{O} 1)$ For all $x, y \in L^{*}$, since $O$ is commutative, so $O\left(x_{1}, y_{1}\right)=O\left(y_{1}, x_{1}\right), O\left(1-x_{2}, 1-\right.$ $\left.y_{2}\right)=O\left(1-y_{2}, 1-x_{2}\right)$, it follows that

$\widetilde{O}(x, y)=\left\langle O\left(x_{1}, y_{1}\right), 1-O\left(1-x_{2}, 1-y_{2}\right)\right\rangle=\left\langle O\left(y_{1}, x_{1}\right), 1-O\left(1-y_{2}, 1-x_{2}\right)\right\rangle=\widetilde{O}(y, x)$.

$(\widetilde{O} 2)$ For all $x, y \in L^{*}$, according to the Boundary condition of $O$, we can get to know $O\left(x_{1}, y_{1}\right)=0 \Leftrightarrow x_{1}=0$ or $y_{1}=0, O\left(1-x_{2}, 1-y_{2}\right)=0 \Leftrightarrow x_{2}=1$ or $y_{2}=1$, it follows that

$\widetilde{O}(x, y)=0_{L^{*}} \Leftrightarrow\left\langle O\left(x_{1}, y_{1}\right), 1-O\left(1-x_{2}, 1-y_{2}\right)\right\rangle=0_{L^{*}} \Leftrightarrow O\left(x_{1}, y_{1}\right)=0,1-O(1-$ $\left.x_{2}, 1-y_{2}\right)=1 \Leftrightarrow x=0_{L^{*}}$ or $y=0_{L^{*}}$.

$(\widetilde{O} 3)$ For all $x, y \in L^{*}$, according to the Boundary condition of $O$, we can get to know $O\left(x_{1}, y_{1}\right)=1 \Leftrightarrow x_{1}=y_{1}=1, O\left(1-x_{2}, 1-y_{2}\right)=1 \Leftrightarrow x_{2}=y_{2}=0$, it follows that

$\widetilde{O}(x, y)=1_{L^{*}} \Leftrightarrow\left\langle O\left(x_{1}, y_{1}\right), 1-O\left(1-x_{2}, 1-y_{2}\right)\right\rangle=1_{L^{*}} \Leftrightarrow O\left(x_{1}, y_{1}\right)=1,1-O(1-$ $\left.x_{2}, 1-y_{2}\right)=0 \Leftrightarrow x=y=1_{L^{*}}$.

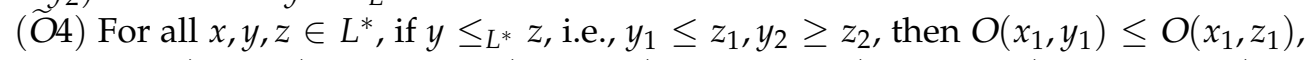
$O\left(1-x_{2}, 1-y_{2}\right) \leq O\left(1-x_{2}, 1-z_{2}\right), 1-O\left(1-x_{2}, 1-y_{2}\right) \geq 1-O\left(1-x_{2}, 1-z_{2}\right)$, it follows that

$\widetilde{O}(x, y)=\left\langle O\left(x_{1}, y_{1}\right), 1-O\left(1-x_{2}, 1-y_{2}\right)\right\rangle \leq_{L^{*}}\left\langle O\left(x_{1}, z_{1}\right), 1-O\left(1-x_{2}, 1-z_{2}\right)\right\rangle=\widetilde{O}(x, z)$.

$(\widetilde{O} 5)$ Firstly, we prove left continuous, i.e., $\widetilde{O}\left(x, \vee_{i \in I} y_{i}\right)=\vee_{i \in I} \widetilde{O}\left(x, y_{i}\right)$ where $x=$ $\left(x_{1}, x_{2}\right), y=\left(y_{1}, y_{2}\right)$. Because the overlap function $O$ is continuous, $O\left(x_{1}, \vee_{i \in I} y_{i 1}\right)=$ $\vee_{i \in I} O\left(x_{1}, y_{i 1}\right)$ and $O\left(1-x_{2}, 1-\wedge_{i \in I} y_{i 2}\right)=O\left(1-x_{2}, \vee_{i \in I}\left(1-y_{i 2}\right)\right)=\vee_{i \in I} O\left(1-x_{2}, 1-\right.$ $\left.y_{i 2}\right)$ is holding.

Then we have

$\widetilde{O}\left(x, \vee_{i \in I} y_{i}\right)=\left\langle O\left(x_{1}, \vee_{i \in I} y_{i 1}\right), 1-O\left(1-x_{2}, 1-\wedge_{i \in I} y_{i 2}\right\rangle=\left\langle O\left(x_{1}, \vee_{i \in I} y_{i 1}\right), 1-O\left(1-x_{2}, \vee_{i \in I}\left(1-y_{i 2}\right)\right)\right\rangle=\left\langle\vee_{i \in I} O\left(x_{1}, y_{i 1}\right)\right.\right.$, $\left.1-\vee_{i \in I} O\left(1-x_{2},\left(1-y_{i 2}\right)\right)\right\rangle=\left\langle\vee_{i \in I} O\left(x_{1}, y_{i 1}\right), \wedge_{i \in I}\left(1-O\left(1-x_{2},\left(1-y_{i 2}\right)\right)\right\rangle=\vee_{i \in I}\left\langle O\left(x_{1}, y_{i 1}\right), 1-O\left(1-x_{2},\left(1-y_{i 2}\right)\right\rangle\right.\right.$ $=\vee_{i \in I} \widetilde{O}\left(x, y_{i}\right)$.

Therefore the function $\widetilde{O}$ is left continuous.

Similarly, we can obtain $\widetilde{O}\left(x, \wedge_{i \in I} y_{i}\right)=\wedge_{i \in I} \widetilde{O}\left(x, y_{i}\right)$, therofore the function $\widetilde{O}$ is right continuous.

Hence the function $\widetilde{O}$ is continuous.

Example 1. Define functions as follows: for $x=\left(x_{1}, x_{2}\right), y=\left(y_{1}, y_{2}\right)$,

(1) $\widetilde{O}(x, y)=\left\langle\min \left(x_{1}, y_{1}\right) \min \left(x_{1}^{2}, y_{1}^{2}\right), \max \left(x_{2}, y_{2}\right) \max \left(x_{2}^{2}, y_{2}^{2}\right)\right\rangle$.

(2) $\widetilde{O}(x, y)=\left\langle\min \left(\sqrt{x_{1}}, \sqrt{y_{1}}\right), \max \left(\sqrt{x_{2}}, \sqrt{y_{2}}\right)\right\rangle$.

(3) $\widetilde{O}(x, y)=\left\langle x_{1} y_{1}, 1-\left(1-x_{2}\right)\left(1-y_{2}\right)\right\rangle$.

(4) $\widetilde{O}(x, y)=\left\langle x_{1} y_{1} \frac{x_{1}+y_{1}}{2}, 1-\left(1-x_{2}\right)\left(1-y_{2}\right) \frac{2-x_{2}-y_{2}}{2}\right\rangle$.

(5) $\widetilde{O}(x, y)=\left\langle 0.5 x_{1} y_{1}+0.5 \max \left(0, x_{1}+y_{1}-1\right), \min \left(1, x_{2}+1-y_{1}, y_{2}+1-x_{1}\right)\right\rangle$.

It is easy to verify that the above functions are IF-overlap function.

Definition 13. Let $\widetilde{O}: L^{*} \times L^{*} \rightarrow L^{*}$ be an IF-overlap function, defined the function $R_{\widetilde{O}}$ : $L^{*} \times L^{*} \rightarrow L^{*}$ as follows:

$$
R_{\widetilde{O}}(x, y)=\sup \left\{z \in L^{*} \mid \widetilde{O}(x, z) \leq_{L^{*}} y\right\}
$$

then $R_{\widetilde{O}}$ is called the residual implication induced by the IF-overlap function $\widetilde{O}$.

Example 2. Define functions as follows: for $x=\left\langle x_{1}, x_{2}\right), y=\left(y_{1}, y_{2}\right\rangle$, 
(1)

$$
R_{\widetilde{O}}(x, y)=\left\{\begin{aligned}
\langle 1,0\rangle & x_{1}^{3} \leq y_{1} \text { and } x_{2}^{3} \leq y_{2} \\
\left\langle 1-y_{2}^{1 / 3}, y_{2}^{1 / 3}\right\rangle & x_{1}^{3} \leq y_{1} \text { and } x_{2}^{3}<y_{2} \\
\left\langle y_{1}^{1 / 3}, 0\right\rangle & x_{1}^{3}>y_{1} \text { and } x_{2}^{3} \geq y_{2} \\
\left\langle y_{1}^{1 / 3}, y_{2}^{1 / 3}\right\rangle & x_{1}^{3}>y_{1} \text { and } x_{2}^{3}<y_{2}
\end{aligned}\right.
$$

$R_{\widetilde{O}}$ is the residual implication induced by the IF-overlap function $\widetilde{O}(x, y)$ in Example (1(1)).

(2)

$$
R_{\widetilde{O}}(x, y)=\left\{\begin{aligned}
\langle 1,0\rangle & x_{1} \leq y_{1}^{2} \text { and } x_{2} \leq y_{2}^{2} \\
\left\langle 1-y_{2}^{2}, y_{2}^{2}\right\rangle & x_{1} \leq y_{1}^{2} \text { and } x_{2}<y_{2}^{2} \\
\left\langle y_{1}^{2}, 0\right\rangle & x_{1}>y_{1}^{2} \text { and } x_{2} \geq y_{2}^{2} \\
\left\langle y_{1}^{2}, y_{2}^{2}\right\rangle & x_{1}>y_{1}^{2} \text { and } x_{2}<y_{2}^{2}
\end{aligned}\right.
$$

$R_{\widetilde{O}}$ is the residual implication induced by the IF-overlap function $\widetilde{O}(x, y)$ in Example (1(2)).

(3) $R_{\widetilde{O}}(x, y)=\left\langle\frac{y_{1}}{x_{1}}, 1-\frac{1-y_{2}}{1-x_{2}}\right\rangle, R_{\widetilde{O}}$ is the residual implication induced by the IF-overlap function $\widetilde{O}(x, y)$ in Example (1(3)).

Definition 14. There exist two overlap functions $O_{1}, O_{2}$, if $O_{1} \leq O_{2}$, defined function as follows: for $x=\left(x_{1}, x_{2}\right), y=\left(y_{1}, y_{2}\right)$,

$$
\widetilde{O}\left(\left(x_{1}, x_{2}\right),\left(y_{1}, y_{2}\right)\right)=\left\langle O_{1}\left(x_{1}, y_{1}\right), 1-O_{2}\left(1-x_{2}, 1-y_{2}\right)\right\rangle
$$

then $\widetilde{O}$ is called representable IF-overlap function.

Example 3. (1) The function $\widetilde{O}(x, y)=\left\langle x_{1} y_{1}, 1-\left(1-x_{2}\right)\left(1-y_{2}\right)\right\rangle$ is a representable IF-overlap function.

(2) $\widetilde{O}(x, y)=\left\langle 0.5 x_{1} y_{1}+0.5 \max \left(0, x_{1}+y_{1}-1\right), \min \left(1, x_{2}+1-y_{1}, y_{2}+1-x_{1}\right)\right\rangle$ is a unrepresentable IF-overlap function.

Let $O_{1}\left(x_{1}, y_{1}\right)=0.5 x_{1} y_{1}+0.5 \max \left(0, x_{1}+y_{1}+1\right), O_{2}\left(x_{2}, y_{2}\right)=1-\min \left(1,2-x_{2}-\right.$ $\left.x_{0}, 2-y_{2}-y_{0}\right)$, where $x_{0}, y_{0} \in[0,1]$ is contant.

$$
O_{2}(1,1)= \begin{cases}x_{0} & x_{0}>y_{0} \\ y_{0} & x_{0} \leq y_{0}\end{cases}
$$

Obviously, $O_{2}(1,1) \neq 1$, that is not satisfied the conditions for overlap function. So, the function $\widetilde{O}(x, y)=\left\langle 0.5 x_{1} y_{1}+0.5 \max \left(0, x_{1}+y_{1}-1\right), \min \left(1, x_{2}+1-y_{1}, y_{2}+1-x_{1}\right)\right\rangle$ is a unrepresentable IF-overlap function.

It is proved that Example 3(2) satisfies the condition of IF-overlap function, but it does not meet the condition of representable IF-overlap function, so the function $\widetilde{O}(x, y)=$ $\left\langle 0.5 x_{1} y_{1}+0.5 \max \left(0, x_{1}+y_{1}-1\right), \min \left(1, x_{2}+1-y_{1}, y_{2}+1-x_{1}\right)\right\rangle$ is a unrepresentable IF-overlap function. For this type of function, we give a more general function expression, i.e., $\widetilde{O}(x, y)=\left\langle\alpha x_{1} y_{1}+(1-\alpha) \max \left(0, x_{1} y_{1}-1\right), \min \left(1, x_{2}+1-y_{1}, y_{2}+1-x_{1}\right)\right\rangle$, where $\alpha \in[0,1]$.

Proposition 2. The function $\widetilde{O}: L^{*} \times L^{*} \rightarrow L^{*}$ is an IF-overlap function if and only if there exist two functions $f, g:[0,1] \times[0,1] \rightarrow[0,1]$ such that

$$
\begin{aligned}
& \forall x=\left(x_{1}, x_{2}\right), y=\left(y_{1}, y_{2}\right) \in L^{*}, \widetilde{O}(x, y)=\left\langle\frac{f\left(x_{1}, y_{1}\right)}{f\left(x_{1}, y_{1}\right)+g\left(x_{1}, y_{1}\right)}, 1-\frac{f\left(1-x_{2}, 1-y_{2}\right)}{f\left(1-x_{2}, 1-y_{2}\right)+g\left(1-x_{2}, 1-y_{2}\right)}\right\rangle \\
& \text { where } \\
& \begin{aligned}
\text { 1. } f \text { and } g \text { are symmetric; } \\
\text { 2. } f \text { is non-decreasing and } g \text { is non-increasing; } \\
\text { 3. } f(x, y)=0 \text { if and only if } x y=0 ; \\
\text { 4. } g(x, y)=0 \text { if and only if } x y=1 ;
\end{aligned}
\end{aligned}
$$




\section{5. $f$ and $g$ are continuous.}

In other words, the IF-overlap function $\widetilde{O}$ can be generated by both $f$ and $g$.

Example 4. (1) Let $f=x y$ and $g=1-x y$, the IF-overlap function generated by $f$ and $g$ is $\widetilde{O}(x, y)=\left\langle x_{1} y_{1}, 1-\left(1-x_{2}\right)\left(1-y_{2}\right)\right\rangle$.

(2) Let $f=x y(x+y)$ and $g=2-x y(x+y)$, the IF-overlap function generated by $f$ and $g$ is $\widetilde{O}(x, y)=\left\langle x_{1} y_{1} \frac{x_{1}+y_{1}}{2}, 1-\left(1-x_{2}\right)\left(1-y_{2}\right) \frac{2-x_{2}-y_{2}}{2}\right\rangle$.

\section{IF-Rough Sets Model Base on IF-Overlap Functions}

In order to popularize the application of IF-rough set model, a new class of IF-rough set model is proposed by combining IF-overlap function. In this section, we will introduce the definition and some properties of this new IF-rough sets model.

Definition 15. Let $\widetilde{O}$ be an IF-overlap function, $R_{\widetilde{O}}$ be an residual implication, and $R$ be an IF similarity relation in $U$, the $(U, R)$ is called IF approximation space. For any IF set $A$ in $U$, the lower and upper approximation of $A$ are the IF sets $R \downarrow_{R_{\tilde{O}}} A$ and $R \uparrow_{\widetilde{O}} A$ is defined by:

$$
\begin{aligned}
R \downarrow_{R_{\tilde{O}}} A(y) & =\inf _{x \in U} R_{\widetilde{O}}(R(x \cdot y), A(x)) \\
R \uparrow_{\widetilde{O}} A(y) & =\sup _{x \in U} \widetilde{O}(R(x \cdot y), A(x))
\end{aligned}
$$

for all $y$ in $U$.

$A$ is called definable if and only if $R \downarrow_{R_{\tilde{O}}} A=R \uparrow_{\tilde{O}} A$. Conversely, called the couple $\left(R \downarrow_{R_{\tilde{O}}} A, R \uparrow_{\tilde{O}} A\right)$ is IF-rough sets, and $R \downarrow_{R_{\tilde{O}}} A$ and $R \uparrow_{\tilde{O}} A$ respectively are referred to as approximation operators under IF and approximation operators above IF.

Next, give an example of IF set, and use IF-rough set based on IF-overlap functions to calculate its lower and upper approximation.

Example 5. Let the $A$ is an IF set, i.e., $A=\frac{\langle 0.8,0.1\rangle}{x_{1}}+\frac{\langle 0.7,0.2\rangle}{x_{2}}+\frac{\langle 0.6,0.1\rangle}{x_{3}}+\frac{\langle 0.9,0.1\rangle}{x_{4}}+\frac{\langle 0.8,0.2\rangle}{x_{5}}, R$ is an IF relation showed in Table 1.

Table 1. IF relation $R$.

\begin{tabular}{cccccc}
\hline $\boldsymbol{R}$ & $x_{\mathbf{1}}$ & $\boldsymbol{x}_{\mathbf{2}}$ & $x_{3}$ & $x_{4}$ & $x_{5}$ \\
\hline$R\left(x_{1}\right)$ & $\langle 0.9,0.0\rangle$ & $\langle 0.7,0.1\rangle$ & $\langle 0.6,0.2\rangle$ & $\langle 0.5,0.1\rangle$ & $\langle 0.3,0.2\rangle$ \\
$R\left(x_{2}\right)$ & $\langle 0.8,0.1\rangle$ & $\langle 0.4,0.4\rangle$ & $\langle 0.8,0.1\rangle$ & $\langle 0.7,0.1\rangle$ & $\langle 1.0,0.0\rangle$ \\
$R\left(x_{3}\right)$ & $\langle 0.7,0.2\rangle$ & $\langle 0.3,0.1\rangle$ & $\langle 0.0,0.6\rangle$ & $\langle 0.2,0.2\rangle$ & $\langle 0.6,0.2\rangle$ \\
$R\left(x_{4}\right)$ & $\langle 0.6,0.1\rangle$ & $\langle 0.5,0.5\rangle$ & $\langle 0.4,0.4\rangle$ & $\langle 0.7,0.2\rangle$ & $\langle 0.3,0.4\rangle$ \\
$R\left(x_{5}\right)$ & $\langle 0.9,0.0\rangle$ & $\langle 0.0,1.0\rangle$ & $\langle 0.1,0.1\rangle$ & $\langle 0.5,0.5\rangle$ & $\langle 0.3,0.3\rangle$ \\
\hline
\end{tabular}

By the Definition 15,let's take $\widetilde{O}$ and $R_{\widetilde{O}}$ for example 1 and 2 , then calculate the lower and upper approximation as follows:

$$
\begin{aligned}
& R \downarrow_{R_{\tilde{O}}} A=\frac{\langle 0.4152,0.5848\rangle}{x_{1}}+\frac{\langle 0.4152,0.5848\rangle}{x_{2}}+\frac{\langle 0.4152,0.5848\rangle}{x_{3}}+\frac{\langle 0.4152,0.5848\rangle}{x_{4}}+\frac{\langle 0.4152,0.5848\rangle}{x_{5}} ; \\
& R \uparrow_{\widetilde{O}} A=\frac{\langle 0.512,0.0001\rangle}{x_{1}}+\frac{\langle 0.343,0.001\rangle}{x_{2}}+\frac{\langle 0.343,0.008\rangle}{x_{3}}+\frac{\langle 0.343,0.008\rangle}{x_{4}}+\frac{\langle 0.343,0.008\rangle}{x_{5}} .
\end{aligned}
$$

The following, we list the properties of intuitionistic fuzzy upper and lower approximation operators, and give concrete examples to show that their idempotent propertie is not set up.

Proposition 3. Let $\left(U, R, \widetilde{O}, R_{\widetilde{O}}\right)$ be an IF approximation space, where $\widetilde{O}$ is an IF-overlap function and $R_{\widetilde{O}}$ is a residual implicator of $\widetilde{O}$. Then for all $A, B \in I F S, R_{1} \subseteq R_{2}$, the following properties hold: 
(1) $A \subseteq B \Rightarrow R \downarrow_{R_{\tilde{O}}} A \subseteq R \downarrow_{R_{\tilde{O}}} B ; R \uparrow_{\widetilde{O}} A \subseteq R \uparrow_{\widetilde{O}} B$;

(2) $R_{1} \downarrow_{R_{\tilde{O}}} A \supseteq R_{2} \downarrow_{R_{\tilde{O}}} A ; R_{1} \uparrow_{\widetilde{O}} A \subseteq R_{2} \uparrow_{\tilde{O}} A$;

(3) $R \downarrow_{R_{\tilde{O}}}(A \cap B)=R \downarrow_{R_{\tilde{O}}} A \cap R \downarrow_{R_{\tilde{O}}} B$;

(4) $R \uparrow_{\widetilde{O}}(A \cup B)=R \uparrow_{\widetilde{O}} A \cup \uparrow_{\widetilde{O}} B$;

(5) $R \downarrow_{R_{\tilde{O}}}(A \cup B) \supseteq R \downarrow_{R_{\tilde{O}}} A \cup R \downarrow_{R_{\tilde{O}}} B$;

(6) $R \uparrow_{\widetilde{O}}(A \cap B) \subseteq R \uparrow_{\widetilde{O}} A \cap \uparrow_{\widetilde{O}} B$.

Proof. (1) It can be directly followed from Definitions 12 and 15.

(2) By the definition of IF relation, if $R_{1} \subseteq R_{2}$ then $R_{1}(x, y) \leq R_{2}(x, y)$, by the Definition 3.1, we have $\widetilde{O}\left(R_{1}(x, y), A(x)\right) \leq \widetilde{O}\left(R_{2}(x, y), A(x)\right)$, then

$$
\sup _{x \in U} \widetilde{O}\left(R_{1}(x \cdot y), A(x)\right) \leq \sup _{x \in U} \widetilde{O}\left(R_{2}(x \cdot y), A(x)\right) .
$$

That is $R_{1} \uparrow_{\widetilde{O}} A \subseteq R_{2} \uparrow_{\widetilde{O}} A$ holds. Similarly, we can verity that $R_{1} \subseteq R_{2}$, then $R_{1} \downarrow_{R_{\tilde{O}}} A \supseteq R_{2} \downarrow_{R_{\tilde{O}}} A$ holds.

(3) By definition to know,

$R \downarrow_{R_{\tilde{O}}}(A \cap B)(y)=\inf _{x \in U} R_{\widetilde{O}}(R(x, y), A(x) \wedge B(x))=\inf _{x \in U} R_{\widetilde{O}}(R(x, y), A(x)) \wedge$ $\inf _{x \in U} R_{\widetilde{O}}(R(x, y), B(x))=R \downarrow_{R_{\tilde{O}}} A(y) \cap R \downarrow_{R_{\tilde{O}}} B(y)$.

(4) By definition to know,

$R \uparrow_{\widetilde{O}}(A \cup B)(y)=\sup _{x \in U} \widetilde{O}(R(x, y), A(x) \vee B(x))=\sup _{x \in U} \widetilde{O}(R(x, y), A(x) \vee$ $\sup _{x \in U} \widetilde{O}\left(R(x, y), B(x)=R \uparrow_{\widetilde{O}} A(y) \cup R \uparrow_{\widetilde{O}} B(y)\right.$.

(5) That can be directly followed from Definition 15 and Propositions 1-4, respectively.

(6) It can be directly followed from Definition 15 and Propositions 1-4, respectively.

In particular, we illustrate that the model is not idempotent, i.e.,

$$
\begin{gathered}
R \uparrow_{\widetilde{O}} A \neq R \uparrow_{\widetilde{O}}\left(R \uparrow_{\widetilde{O}} A\right) \\
R \downarrow_{R_{\widetilde{O}}} A \neq R \downarrow_{R_{\widetilde{O}}}\left(R \downarrow_{R_{\widetilde{O}}} A\right)
\end{gathered}
$$

Example 6. Let $A$ be an IF sets, $A=\frac{\langle 0.3,0.5\rangle}{x_{1}}+\frac{\langle 0.4,0.6\rangle}{x_{2}}+\frac{\langle 0.5,0.5\rangle}{x_{3}}+\frac{\langle 0.7,0.2\rangle}{x_{4}}+\frac{\langle 0.8,0.1\rangle}{x_{5}}$, and $R$ be an IF relation as Table 1, then by calculating, we have the results as follows: $R \uparrow_{\widetilde{O}}$ $A=\frac{\langle 0.512,0.0001\rangle}{x_{1}}+\frac{\langle 0.125,0.125\rangle}{x_{2}}+\frac{\langle 0.064,0.064\rangle}{x_{3}}+\frac{\langle 0.343,0.008\rangle}{x_{4}}+\frac{\langle 0.125,0.125\rangle}{x_{5}} ; R \uparrow_{\widetilde{O}}\left(R \uparrow_{\widetilde{O}} A\right)=$ $\frac{\langle 0.1342,0.0\rangle}{x_{1}}+\frac{\langle 0.1342,0.001\rangle}{x_{2}}+\frac{\langle 0.1342,0.008\rangle}{x_{3}}+\frac{\langle 0.125,0.001\rangle}{x_{4}}+\frac{\langle 0.027,0.008\rangle}{x_{5}}$. A).

Obviously, $R \uparrow_{\widetilde{O}} A \neq R \uparrow_{\widetilde{O}}\left(R \uparrow_{\widetilde{O}} A\right)$, by calculation know $R \downarrow_{R_{\tilde{O}}} A \neq R \downarrow_{R_{\tilde{O}}}\left(R \downarrow_{R_{\tilde{O}}}\right.$

\section{Application Example}

In this section, we will describe the application of the new IF-rough set model to MCDM (multi-criteria decision making) problems, and compares the decision results with other models.

\subsection{Problem Description}

In a public company, shareholders want to elect an executive director who have both ability and political integrity, in order to create more value for the company. Let $X=\left\{x_{i}\right.$ : $\mathrm{i}=1,2, \ldots, \mathrm{n}\}$ be the universe of $n$ alternatives, $C=\left\{C_{j}: \mathrm{j}=1,2, \ldots, \mathrm{m}\right\}$ be the set of $m$ criteria. $C_{j}\left(x_{i}\right)=\left(\mu\left(x_{i}\right), v\left(x_{i}\right)\right)$ where $0 \leq \mu\left(x_{i}\right)+v\left(x_{i}\right) \leq 1, \mu\left(x_{i}\right), v\left(x_{i}\right)$ are the degrees of membership and non-membership of $C_{j}\left(x_{i}\right)$, respectively. $C_{j}\left(x_{i}\right)$ denotes the ability value of the alternative $x_{i}$ to the criterion $C_{j}$ given a lot of judges. Assuming that for any alternative $x_{i}$, there is at least one criterion $C_{j}$ such that the value of the alternative $x_{i}$ for the criterion $C_{j}$ is equal to $\langle 1,0\rangle$. In the following, we can solve the decision-making problem by 
means of the principle of the IF TOPSIS methods and the IF-rough set models. We apply the algorithm in recruiting alternative in a public company to choose the best director.

\subsection{Decision-Making Method}

Firstly, we build one IF set $C_{j}$ is a description of $X$ given by a lot of experts through their experience, then give the IF relation $R$ for each $x_{i}$.

Secondly, by the IF relation $\mathrm{R}$, the positive ideal solution and the negative ideal solution of the alternative $x_{i}$ are defined as:

$$
A^{+}\left(x_{i}\right)=\left(\mu_{A^{+}}\left(x_{i}\right), v_{A^{+}}\left(x_{i}\right)\right)
$$

where $\mu_{A^{+}}\left(x_{i}=\max _{j=1}^{m}\left(\mu_{C_{j}}\left(x_{i}\right)\right), v_{A^{+}}\left(x_{i}=\min _{j=1}^{m}\left(v_{C_{j}}\left(x_{i}\right)\right)\right.\right.$;

$$
A^{-}\left(x_{i}\right)=\left(\mu_{A^{-}}\left(x_{i}\right), v_{A^{-}}\left(x_{i}\right)\right)
$$

where $\mu_{A^{-}}\left(x_{i}=\min _{j=1}^{m}\left(\mu_{C_{j}}\left(x_{i}\right)\right), v_{A^{-}}\left(x_{i}=\max _{j=1}^{m}\left(v_{C_{j}}\left(x_{i}\right)\right)\right.\right.$.

We can receive that the positive ideal solution $A^{+}$and the negative ideal solution $A^{-}$. Where $A^{+}, A^{-} \in \operatorname{IF}(U)$. Thirdly, we compute the IF rough approximation of $A^{+}$and $A^{-}$ by the IF-rough sets. Then there are two types calculating, by the score function $L(A)\left(x_{i}\right)=$ $\mu_{A}\left(x_{i}\right)+v_{A}\left(x_{i}\right) \cdot \pi_{A}\left(x_{i}\right)$ where $\pi_{A}\left(x_{i}\right)=1-\mu_{A}\left(x_{i}\right)-v_{A}\left(x_{i}\right)$ and the summation formula of IF sets $\tilde{x} \oplus \tilde{y}=\left\langle\mu_{\tilde{x}}+\mu_{\tilde{y}}-\mu_{\tilde{x}} \mu_{\tilde{y}}, v_{\tilde{x}} v_{\tilde{y}}\right\rangle$ where $\tilde{x}=\left\langle\mu_{\tilde{x}}, v_{\tilde{x}}\right\rangle, \tilde{y}=\left\langle\mu_{\tilde{y}}, v_{\tilde{y}}\right\rangle, \tilde{x}, \tilde{y} \in D^{*}$ and $\lambda \tilde{x}=\left\langle 1-(1-\mu \tilde{x})^{\lambda}, v_{\tilde{x}}^{\lambda}\right\rangle, \lambda>0$, then for each $x_{i} \in U$, two ranking functions of $x_{i}$ are defined as:

The first type:

$$
P^{-}\left(x_{i}\right)=L\left(R \downarrow A^{-} \bigoplus R \uparrow A^{-}\right), P^{+}\left(x_{i}\right)=L\left(R \downarrow A^{+} \bigoplus R \uparrow A^{+}\right) ;
$$

The second type:

$$
P^{-}\left(x_{i}\right)=L\left(\alpha R \downarrow A^{-} \bigoplus(1-\alpha) R \uparrow A^{-}\right), P^{+}\left(x_{i}\right)=L\left(\alpha R \downarrow A^{+} \bigoplus(1-\alpha) R \uparrow A^{+}\right) .
$$

where $\alpha \in[0,1]$ be a level adjustment value.

Lastly, based on the principle of the TOPSIS methods, the relative closeness coefficient of every alternative $x_{i}$ about $P^{-}$and $P^{+}$is defined as: $\delta\left(x_{i}\right)=\frac{P^{-}\left(x_{i}\right)}{P^{-}\left(x_{i}\right)+P^{+}\left(x_{i}\right)}$. According to the values of $\delta\left(x_{i}\right)$, we can rank these alternatives. Lastly, through the ranking order of all alternatives, we can choose the best alternative.

\subsection{Algorithm for IF-Rough Sets Models with IF Information}

We come up with an algorithm for IF rough sets models based on MCDM problem with IF information. Now a company want to choose the best one from six candidates. Let $U=\left\{x_{1}, x_{2}, x_{3}, x_{4}, x_{5}, x_{6}\right\}$ be the set of six candidates. Let $C=\left\{C_{1}, C_{2}, C_{3}, C_{4}, C_{5}\right\}$ be five criteria, $C_{1}, C_{2}, C_{3}, C_{4}, C_{5}$ represent emotional quotient, work ability, language expression skills, management ability and resilience ability, respectively. Let $C_{j}\left(x_{i}\right)=$ $\left\langle\mu\left(x_{i}\right), v\left(x_{i}\right)\right\rangle,(j=1,2, \ldots, 5 ; i=1,2, \ldots, 6)$ where $\mu\left(x_{i}\right)$ and $v\left(x_{i}\right)$ are the degrees of the membership and the non-membership of the alternative $x_{i}$ to the criterion $C_{j}$, respectively. Suppose that for each alternative $x_{i}$, there exists the criterion $C_{j}$ such that $C_{j}\left(x_{i}\right)=\langle 1,0\rangle$. The IF relation $R$ based IF rough sets of six alternatives are as Table 2. 
Table 2. IF relation $R$.

\begin{tabular}{ccccccc}
\hline $\mathbf{R}$ & $\boldsymbol{x}_{\mathbf{1}}$ & $\boldsymbol{x}_{\mathbf{2}}$ & $\boldsymbol{x}_{\mathbf{3}}$ & $\boldsymbol{x}_{\mathbf{4}}$ & $\boldsymbol{x}_{\mathbf{5}}$ & $\boldsymbol{x}_{\mathbf{6}}$ \\
\hline $\boldsymbol{R} / \boldsymbol{U}$ & $\boldsymbol{x}_{\mathbf{1}}$ & $\boldsymbol{x}_{\mathbf{2}}$ & $\boldsymbol{x}_{\mathbf{3}}$ & $\boldsymbol{x}_{\mathbf{4}}$ & $\boldsymbol{x}_{\mathbf{5}}$ & $\boldsymbol{x}_{\mathbf{6}}$ \\
\hline$R\left(x_{1}\right)$ & $\langle 1,0\rangle$ & $\langle 0.6,0.4\rangle$ & $\langle 0.6,0.3\rangle$ & $\langle 0.6,0.2\rangle$ & $\langle 0.5,0.3\rangle$ & $\langle 0.5,0.4\rangle$ \\
$R\left(x_{2}\right)$ & $\langle 0.8,0.2\rangle$ & $\langle 1,0\rangle$ & $\langle 0.8,0.2\rangle$ & $\langle 0.8,0.2\rangle$ & $\langle 0.6,0.4\rangle$ & $\langle 0.6,0.4\rangle$ \\
$R\left(x_{3}\right)$ & $\langle 0.7,0.25\rangle$ & $\langle 0.7,0.1\rangle$ & $\langle 1,0\rangle$ & $\langle 0.7,0.3\rangle$ & $\langle 0.5,0.5\rangle$ & $\langle 0.8,0.2\rangle$ \\
$R\left(x_{4}\right)$ & $\langle 0.3,0.7\rangle$ & $\langle 0.3,0.5\rangle$ & $\langle 0.3,0.45\rangle$ & $\langle 1,0\rangle$ & $\langle 0.6,0.2\rangle$ & $\langle 0.6,0.4\rangle$ \\
$R\left(x_{5}\right)$ & $\langle 0.5,0.5\rangle$ & $\langle 0.7,0.3\rangle$ & $\langle 0.7,0.25\rangle$ & $\langle 0.9,0.1\rangle$ & $\langle 1,0\rangle$ & $\langle 0.7,0.3\rangle$ \\
$R\left(x_{6}\right)$ & $\langle 0.7,0.3\rangle$ & $\langle 0.7,0.1\rangle$ & $\langle 0.7,0.15\rangle$ & $\langle 0.7,0.3\rangle$ & $\langle 0.5,0.5\rangle$ & $\langle 1,0\rangle$ \\
\hline
\end{tabular}

Then calculate the positive ideal solution $A^{+}$and the negative ideal solution $A^{-}$ as follows:

$$
\begin{aligned}
& A^{+}=\frac{\langle 1,0\rangle}{x_{1}}+\frac{\langle 1,0\rangle}{x_{2}}+\frac{\langle 1,0\rangle}{x_{3}}+\frac{\langle 1,0\rangle}{x_{4}}+\frac{\langle 1,0\rangle}{x_{5}}+\frac{\langle 1,0\rangle}{x_{6}} ; \\
& A^{-}=\frac{\langle 0.3,0.7\rangle}{x_{1}}+\frac{\langle 0.3,0.5\rangle}{x_{2}}+\frac{\langle 0.3,0.45\rangle}{x_{3}}+\frac{\langle 0.6,0.3\rangle}{x_{4}}+\frac{\langle 0.5,0.5\rangle}{x_{5}}+\frac{\langle 0.5,0.4\rangle}{x_{6}} .
\end{aligned}
$$

We calculate the approximation operator of $A^{+}$and $A^{-}$through three IF-rough sets models, then calculate the $P^{+}\left(x_{i}\right)$ and $P^{-}\left(x_{i}\right)$ for each $x_{i} \in U$,respectively. Last, calculate the $\delta\left(x_{i}\right)$ for each $x_{i} \in U$ and rank for all alternatives.

case 1 IF-rough sets model.

By the definition, we have following results:

$R \downarrow A^{+}=R \uparrow A^{+}=U$;

$R \downarrow A^{-}=\frac{\langle 0.3,0.7\rangle}{x_{1}}+\frac{\langle 0.3,0.7\rangle}{x_{2}}+\frac{\langle 0.3,0.7\rangle}{x_{3}}+\frac{\langle 0.45,0.5\rangle}{x_{4}}+\frac{\langle 0.3,0.5\rangle}{x_{5}}+\frac{\langle 0.3,0.7\rangle}{x_{6}}$;

$R \uparrow A^{-}=\frac{\langle 0.6,0.3\rangle}{x_{1}}+\frac{\langle 0.6,0.3\rangle}{x_{2}}+\frac{\langle 0.6,0.3\rangle}{x_{3}}+\frac{\langle 0.6,0.3\rangle}{x_{4}}+\frac{\langle 0.6,0.3\rangle}{x_{5}}+\frac{\langle 0.6,0.3\rangle}{x_{6}}$.

The first ranking function type, we have the following results:

$P^{+}\left(x_{i}\right)=\frac{1}{x_{1}}+\frac{1}{x_{2}}+\frac{1}{x_{3}}+\frac{1}{x_{4}}+\frac{1}{x_{5}}+\frac{1}{x_{6}} ;$

$P^{-}\left(x_{i}\right)=\frac{0.7347}{x_{1}}+\frac{0.7347}{x_{2}}+\frac{0.7347}{x_{3}}+\frac{0.7905}{x_{4}}+\frac{0.7395}{x_{5}}+\frac{0.7347}{x_{6}}$.

By the formula, we have

$\delta=\frac{0.4235}{x_{1}}+\frac{0.4235}{x_{2}}+\frac{0.4235}{x_{3}}+\frac{0.4415}{x_{4}}+\frac{0.4251}{x_{5}}+\frac{0.4235}{x_{6}}$.

According to the value of $\delta\left(x_{i}\right)$. We rank six alternatives as follows:

$$
x_{4} \succ x_{5} \succ x_{1} \approx x_{2} \approx x_{3} \approx x_{6}
$$

Thus, we can choose the best alternative $x_{4}$.

The second ranking function type,let $\alpha=0.5$, where $\alpha$ is a level adjustment. Then we have the following results:

$$
\begin{aligned}
& P^{+}\left(x_{i}\right)=\frac{1}{x_{1}}+\frac{1}{x_{2}}+\frac{1}{x_{3}}+\frac{1}{x_{4}}+\frac{1}{x_{5}}+\frac{1}{x_{6}} ; \\
& P^{-}\left(x_{i}\right)=\frac{0.5033}{x_{1}}+\frac{0.5033}{x_{2}}+\frac{0.5033}{x_{3}}+\frac{0.5626}{x_{4}}+\frac{0.5258}{x_{5}}+\frac{0.5033}{x_{6}} .
\end{aligned}
$$

By the formula, we have $\delta=\frac{0.3348}{x_{1}}+\frac{0.3348}{x_{2}}+\frac{0.3348}{x_{3}}+\frac{0.36}{x_{4}}+\frac{0.3445}{x_{5}}+\frac{0.3348}{x_{6}}$.

According to the value of $\delta\left(x_{i}\right)$. We rank six alternatives as follows:

$$
x_{4} \succ x_{5} \approx x_{1} \approx x_{2} \approx x_{3} \approx x_{6}
$$

Thus, we can choose the best alternative $x_{4}$.

case $2(I, T)$-IF rough sets model. 
Let $T(x, y)=\left(\min \left(x_{1}, y_{1}\right), \max \left(x_{2}, y_{2}\right)\right)$,

$$
I(x, y)=\left\{\begin{aligned}
\langle 1,0\rangle & x_{1} \leq y_{1} \text { and } x_{2} \geq y_{2} \\
\left(1-y_{2}, y_{2}\right) & x_{1} \leq y_{1} \text { and } x_{2}<y_{2} \\
\left(y_{1}, 0\right) & x_{1}>y_{1} \text { and } x_{2} \geq y_{2} \\
\left(y_{1}, y_{2}\right) & x_{1}>y_{1} \text { and } x_{2}<y_{2}
\end{aligned}\right.
$$

Definition 16 ([35]). Let $\alpha=\left\langle\mu_{\alpha}, v_{\alpha}\right\rangle$ be an IF value, and the score function of the IF value $\alpha$ is defined as follows:

$$
S(\alpha)=\left(\mu_{\alpha}-v_{\alpha}\right)\left(1+\pi_{\alpha}\right)
$$

where $\pi_{\alpha}=1-\mu_{\alpha}-v_{\alpha}$

Definition 17 ([35]). Let $\alpha=\left\langle\mu_{\alpha}, v_{\alpha}\right\rangle$ and $\beta=\left\langle\mu_{\beta}, v_{\beta}\right\rangle$ be two IF values, and $S(\alpha), S(\beta)$ are score function of $\alpha$ and $\beta$ respectively, then

(1) If $S(\alpha)>S(\beta)$, called $\alpha$ is greater than $\beta$, i.e., $\alpha>\beta$;

(2) If $S(\alpha)=S(\beta)$, then,

if $\mu_{\alpha}>\mu_{\beta}$, called $\alpha$ is greater than $\beta$, i.e., $\alpha>\beta$;

if $\mu_{\alpha}<\mu_{\beta}$, called $\alpha$ is less than $\beta$, i.e., $\alpha<\beta$.

$B y$ the definition, we have following results:

$R \downarrow_{I} A^{+}=R \uparrow_{T} A^{+}=U$;

$R \downarrow_{I} A^{-}=\frac{\langle 0.3,0.7\rangle}{x_{1}}+\frac{\langle 0.3,0.7\rangle}{x_{2}}+\frac{\langle 0.3,0.7\rangle}{x_{3}}+\frac{\langle 0.3,0.7\rangle}{x_{4}}+\frac{\langle 0.3,0.7\rangle}{x_{5}}+\frac{\langle 0.3,0.7\rangle}{x_{6}} ;$

$R \uparrow_{T} A^{-}=\frac{\langle 0.5,0.4\rangle}{x_{1}}+\frac{\langle 0.5,0.4\rangle}{x_{2}}+\frac{\langle 0.5,0.4\rangle}{x_{3}}+\frac{\langle 0.6,0.3\rangle}{x_{4}}+\frac{\langle 0.6,0.4\rangle}{x_{5}}+\frac{\langle 0.6,0.4\rangle}{x_{6}}$.

The first ranking function type, we have the following results:

$P^{+}\left(x_{i}\right)=\frac{1}{x_{1}}+\frac{1}{x_{2}}+\frac{1}{x_{3}}+\frac{1}{x_{4}}+\frac{1}{x_{5}}+\frac{1}{x_{6}} ;$

$P^{-}\left(x_{i}\right)=\frac{0.6696}{x_{1}}+\frac{0.6696}{x_{2}}+\frac{0.6696}{x_{3}}+\frac{0.7347}{x_{4}}+\frac{0.72}{x_{5}}+\frac{0.72}{x_{6}} ;$

$\delta=\frac{0.4011}{x_{1}}+\frac{0.4011}{x_{2}}+\frac{0.4011}{x_{3}}+\frac{0.4235}{x_{4}}+\frac{0.4186}{x_{5}}+\frac{0.4186}{x_{6}}$.

$$
x_{4} \succ x_{5} \approx x_{6} \succ x_{1} \approx x_{2} \approx x_{3}
$$

Thus, we can choose the best alternative $x_{4}$.

The second ranking function type, let $\alpha=0.5$, where $\alpha$ is a level adjustment. Then we have the following results:

$$
\begin{aligned}
& P^{+}\left(x_{i}\right)=\frac{1}{x_{1}}+\frac{1}{x_{2}}+\frac{1}{x_{3}}+\frac{1}{x_{4}}+\frac{1}{x_{5}}+\frac{1}{x_{6}} ; \\
& P^{-}\left(x_{i}\right)=\frac{0.4414}{x_{1}}+\frac{0.4414}{x_{2}}+\frac{0.4414}{x_{3}}+\frac{0.5033}{x_{4}}+\frac{0.4708}{x_{5}}+\frac{0.4708}{x_{6}} ; \\
& \delta=\frac{0.3062}{x_{1}}+\frac{0.3062}{x_{2}}+\frac{0.3062}{x_{3}}+\frac{0.3348}{x_{4}}+\frac{0.3201}{x_{5}}+\frac{0.3201}{x_{6}} . \\
& \quad x_{4} \succ x_{5} \approx x_{6} \succ x_{1} \approx x_{2} \approx x_{3}
\end{aligned}
$$

Thus, we can choose the best alternative $x_{4}$. 
case $3\left(R_{\widetilde{O}}, \widetilde{O}\right)$-IF rough sets model.

Let $\widetilde{O}(x, y)=\left\langle\min \left(x_{1}, y_{1}\right) \min \left(x_{1}^{2}, y_{1}^{2}\right), \max \left(x_{2}, y_{2}\right) \max \left(x_{2}^{2}, y_{2}^{2}\right)\right.$

$$
R_{\widetilde{O}}(x, y)=\left\{\begin{array}{rr}
\langle 1,0\rangle & x_{1}^{3} \leq y_{1} \text { and } x_{2}^{3} \leq y_{2} \\
\left(1-y_{2}^{1 / 3}, y_{2}^{1 / 3}\right) & x_{1}^{3} \leq y_{1} \text { and } x_{2}^{3}<y_{2} \\
\left(y_{1}^{1 / 3}, 0\right) & x_{1}^{3}>y_{1} \text { and } x_{2}^{3} \geq y_{2} \\
\left(y_{1}^{1 / 3}, y_{2}^{1 / 3}\right) & x_{1}^{3}>y_{1} \text { and } x_{2}^{3}<y_{2}
\end{array}\right.
$$

By the definition, we have following results:

$R \downarrow_{R_{\tilde{O}}} A^{+}=R \uparrow_{\widetilde{O}} A^{+}=U ;$

$R \downarrow_{R_{\tilde{O}}} A^{-}=\frac{\langle 0.2063,0.7937\rangle}{x_{1}}+\frac{\langle 0.1121,0.8879\rangle}{x_{2}}+\frac{\langle 0.1121,0.8879\rangle}{x_{3}}+\frac{\langle 0.1121,0.8879\rangle}{x_{4}}+\frac{\langle 0.1121,0.8879\rangle}{x_{5}}+$ $\frac{\langle 0.1121,0.8879\rangle}{x_{6}}$;

$R \uparrow_{\widetilde{O}} A^{-}=\frac{\langle 0.125,0.064\rangle}{x_{1}}+\frac{\langle 0.125,0.064\rangle}{x_{2}}+\frac{\langle 0.125,0.064\rangle}{x_{3}}+\frac{\langle 0.216,0.064\rangle}{x_{4}}+\frac{\langle 0.216,0.027\rangle}{x_{5}}+\frac{\langle 0.216,0.027\rangle}{x_{6}}$.

The first ranking function type, we have the following results:

$P^{+}\left(x_{i}\right)=\frac{1}{x_{1}}+\frac{1}{x_{2}}+\frac{1}{x_{3}}+\frac{1}{x_{4}}+\frac{1}{x_{5}}+\frac{1}{x_{6}} ;$

$P^{-}\left(x_{i}\right)=\frac{0.3382}{x_{1}}+\frac{0.2597}{x_{2}}+\frac{0.264}{x_{3}}+\frac{0.3402}{x_{4}}+\frac{0.32}{x_{5}}+\frac{0.32}{x_{6}} ;$

$\delta=\frac{0.2527}{x_{1}}+\frac{0.2062}{x_{2}}+\frac{0.2089}{x_{3}}+\frac{0.2538}{x_{4}}+\frac{0.2424}{x_{5}}+\frac{0.2424}{x_{6}}$.

$$
x_{4} \succ x_{1} \succ x_{5} \approx x_{6} \succ x_{3} \succ x_{2}
$$

Thus, we can choose the best alternative $x_{4}$.

The second ranking function type, let $\alpha=0.5$, where $\alpha$ is a level adjustment. Then we have the following results:

$$
\begin{aligned}
& P^{+}\left(x_{i}\right)=\frac{1}{x_{1}}+\frac{1}{x_{2}}+\frac{1}{x_{3}}+\frac{1}{x_{4}}+\frac{1}{x_{5}}+\frac{1}{x_{6}} ; \\
& P^{-}\left(x_{i}\right)=\frac{0.3037}{x_{1}}+\frac{0.2719}{x_{2}}+\frac{0.2719}{x_{3}}+\frac{0.3027}{x_{4}}+\frac{0.2709}{x_{5}}+\frac{0.2709}{x_{6}} ; \\
& \delta=\frac{0.233}{x_{1}}+\frac{0.2318}{x_{2}}+\frac{0.2318}{x_{3}}+\frac{0.2353}{x_{4}}+\frac{0.2132}{x_{5}}+\frac{0.2132}{x_{6}} . \\
& \quad x_{4} \succ x_{1} \succ x_{2} \approx x_{3} \succ x_{5} \approx x_{6}
\end{aligned}
$$

Thus, we can choose the best alternative $x_{4}$.

case $4\left(R_{\widetilde{O}}, \widetilde{O}\right)$-IF rough sets model.

Now let $\widetilde{O}(x, y)=\left\langle\min \left(\sqrt{x_{1}}, \sqrt{y_{1}}\right), \max \left(\sqrt{x_{2}}, \sqrt{y_{2}}\right)\right\rangle$

$$
R_{\widetilde{O}}(x, y)=\left\{\begin{aligned}
\langle 1,0\rangle & x_{1} \leq y_{1}^{2} \text { and } x_{2} \leq y_{2}^{2} \\
\left\langle 1-y_{2}^{2}, y_{2}^{2}\right\rangle & x_{1} \leq y_{1}^{2} \text { and } x_{2}<y_{2}^{2} \\
\left\langle y_{1}^{2}, 0\right\rangle & x_{1}>y_{1}^{2} \text { and } x_{2} \geq y_{2}^{2} \\
\left\langle y_{1}^{2}, y_{2}^{2}\right\rangle & x_{1}>y_{1}^{2} \text { and } x_{2}<y_{2}^{2}
\end{aligned}\right.
$$

By the definition, we have following results:

$$
\begin{aligned}
& \quad R \downarrow_{R_{\tilde{O}}} A^{+}=R \uparrow_{\widetilde{O}} A^{+}=U ; \\
& \quad R \downarrow_{R_{\tilde{O}}} A^{-}=\frac{\langle 0.09,0.49\rangle}{x_{1}}+\frac{\langle 0.09,0.49\rangle}{x_{2}}+\frac{\langle 0.09,0.49\rangle}{x_{3}}+\frac{\langle 0.09,0.49\rangle}{x_{4}}+\frac{\langle 0.09,0.49\rangle}{x_{5}}+\frac{\langle 0.09,0.49\rangle}{x_{6}} ; \\
& \quad R \uparrow_{\widetilde{O}} A^{-}=\frac{\langle 0.7071,0.6325\rangle}{x_{1}}+\frac{\langle 0.7071,0.6325\rangle}{x_{2}}+\frac{\langle 0.7071,0.6325\rangle}{x_{3}}+\frac{\langle 0.7746,0.5477\rangle}{x_{4}}+\frac{\langle 0.7746,0.5476\rangle}{x_{5}}+ \\
& \frac{\langle 0.7746,0.6325\rangle}{x_{6}} .
\end{aligned}
$$

The first ranking function type, we have the following results: 


$$
\begin{aligned}
& P^{+}\left(x_{i}\right)=\frac{1}{x_{1}}+\frac{1}{x_{2}}+\frac{1}{x_{3}}+\frac{1}{x_{4}}+\frac{1}{x_{5}}+\frac{1}{x_{6}} ; \\
& P^{-}\left(x_{i}\right)=\frac{0.72}{x_{1}}+\frac{0.72}{x_{2}}+\frac{0.72}{x_{3}}+\frac{0.7779}{x_{4}}+\frac{0.7776}{x_{5}}+\frac{0.7624}{x_{6}} ; \\
& \delta=\frac{0.4186}{x_{1}}+\frac{0.4186}{x_{2}}+\frac{0.4186}{x_{3}}+\frac{0.4375}{x_{4}}+\frac{0.4374}{x_{5}}+\frac{0.4326}{x_{6}} . \\
& x_{4} \succ x_{5} \succ x_{6} \succ x_{1} \approx x_{2} \approx x_{3}
\end{aligned}
$$

Thus, we can choose the best alternative $x_{4}$.

The second ranking function type, let $\alpha=0.5$, where $\alpha$ is a level adjustment. Then we have the following results:

$$
\begin{aligned}
& P^{+}\left(x_{i}\right)=\frac{1}{x_{1}}+\frac{1}{x_{2}}+\frac{1}{x_{3}}+\frac{1}{x_{4}}+\frac{1}{x_{5}}+\frac{1}{x_{6}} ; \\
& P^{-}\left(x_{i}\right)=\frac{0.4612}{x_{1}}+\frac{0.4612}{x_{2}}+\frac{0.4612}{x_{3}}+\frac{0.5314}{x_{4}}+\frac{0.5312}{x_{5}}+\frac{0.4893}{x_{6}} ; \\
& \delta=\frac{0.3156}{x_{1}}+\frac{0.3156}{x_{2}}+\frac{0.3156}{x_{3}}+\frac{0.3392}{x_{4}}+\frac{0.3391}{x_{5}}+\frac{0.3285}{x_{6}} . \\
& x_{4} \succ x_{5} \succ x_{6} \succ x_{1} \approx x_{2} \approx x_{3}
\end{aligned}
$$

Thus, we can choose the best alternative $x_{4}$.

\subsection{Comparative Analysis}

In this subsection, first of all, we make a comparison between three models with no level adjustment $\alpha$ with IF information as shown in Table 3. Then a comparison among three models with level adjustment $\alpha=0.5$, results as shown in Table 4 and analyze it.

Table 3. Ranking orders of alternative with no $\alpha$.

\begin{tabular}{lc}
\hline Models & Ranking Orders of Six Alternatives \\
\hline case1 & $x_{4} \succ x_{5} \succ x_{1} \approx x_{2} \approx x_{3} \approx x_{6}$ \\
caes2 & $x_{4} \succ x_{5} \approx x_{6} \succ x_{1} \approx x_{2} \approx x_{3}$ \\
case3 & $x_{4} \succ x_{1} \succ x_{5} \approx x_{6} \succ x_{3} \succ x_{2}$ \\
case4 & $x_{4} \succ x_{5} \succ x_{6} \succ x_{1} \approx x_{2} \approx x_{3}$ \\
\hline
\end{tabular}

Table 4. Ranking orders of alternative with $\alpha=0.5$.

\begin{tabular}{lc}
\hline Models & Ranking Orders of Six Alternatives \\
\hline case1 & $x_{4} \succ x_{5} \approx x_{1} \approx x_{2} \approx x_{3} \approx x_{6}$ \\
caes2 & $x_{4} \succ x_{5} \approx x_{6} \succ x_{1} \approx x_{2} \approx x_{3}$ \\
case3 & $x_{4} \succ x_{1} \succ x_{2} \approx x_{3} \succ x_{5} \approx x_{6}$ \\
case4 & $x_{4} \succ x_{5} \succ x_{6} \succ x_{1} \approx x_{2} \approx x_{3}$ \\
\hline
\end{tabular}

It can be seen from the table that the multi-criteria decision-making method proposed in this consistent with the decision result obtained by existing models, i.e., $x_{4}$ is the best alternative. This phenomenon shows that the model proposed in this paper is effective. Secondly, by the Tables 3 and 4, we can see the model with level adjustment will be better results. Lastly, by the model of case 1, we have almost the same ranking, by the model of case 2 , we can also find $x_{1}, x_{2}, x_{3}$ almost same. Therefore, the two kinds of models cannot make a good ranking in this kind of problems. The model of case 2 is IF rough set based on IF triangular norm, since IF triangular norm satisfies associativity. The IF-overlap function is an extension of IF triangular norm, which does not meet associativity, therefore the model that proposed by this paper has a wider ranger of practical applications and is of effectiveness and application value.

\subsection{Sensitivity Analysis}

Using the similar method in case 1 , let $\alpha=\{0,0.1, \ldots, 0.9,1\}$, we can obtain the results as shown in Table 5. Through this table, we can find that the results are different with different values of $\alpha$. If $\alpha=0$, then the six alternatives have equivalent interest. So, the 
$\alpha=0$ is not perfect when making a decision in real life. When $\alpha \neq 0$, the results of others are same. The best selection is $x_{4}$, respectively.

Table 5. Ranking orders of alternative with different $\alpha$ in case 1.

Different Value of $\alpha$

$\alpha=0$

$\alpha=0.1$

$\alpha=0.2$

$\alpha=0.3$

$\alpha=0.4$

$\alpha=0.5$

$\alpha=0.6$

$\alpha=0.7$

$\alpha=0.8$

$\alpha=0.9$

$\alpha=1$

\section{Ranking Orders of Six Alternatives}

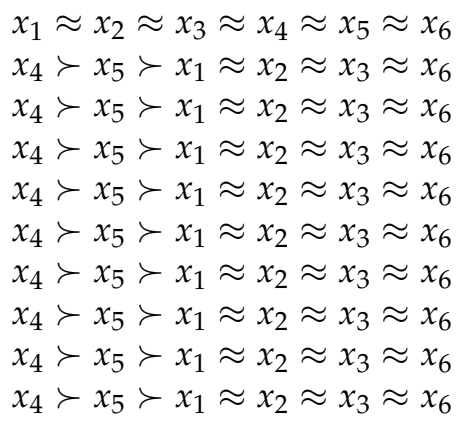

Using the similar method in case 2 , let $\alpha=\{0,0.1, \ldots, 0.9,1\}$, we can obtain the results as shown in Table 6. Through this table, we can find that the results are different with different values of $\alpha$. If $\alpha=1$, then the six alternatives have equivalent interest. So, the $\alpha=1$ is not perfect when making a decision in real life. When $\alpha \neq 1$, the results of others are same. The best selection is $x_{4}$, respectively.

Table 6. Ranking orders of alternative with different $\alpha$ in case 2 .

\section{Different Value of $\alpha$}

$$
\begin{gathered}
\alpha=0 \\
\alpha=0.1 \\
\alpha=0.2 \\
\alpha=0.3 \\
\alpha=0.4 \\
\alpha=0.5 \\
\alpha=0.6 \\
\alpha=0.7 \\
\alpha=0.8 \\
\alpha=0.9 \\
\alpha=1
\end{gathered}
$$

Ranking Orders of Six Alternatives

$$
\begin{aligned}
& x_{4} \succ x_{5} \approx x_{6} \succ x_{1} \approx x_{2} \approx x_{3} \\
& x_{4} \succ x_{5} \approx x_{6} \succ x_{1} \approx x_{2} \approx x_{3} \\
& x_{4} \succ x_{5} \approx x_{6} \succ x_{1} \approx x_{2} \approx x_{3} \\
& x_{4} \succ x_{5} \approx x_{6} \succ x_{1} \approx x_{2} \approx x_{3} \\
& x_{4} \succ x_{5} \approx x_{6} \succ x_{1} \approx x_{2} \approx x_{3} \\
& x_{4} \succ x_{5} \approx x_{6} \succ x_{1} \approx x_{2} \approx x_{3} \\
& x_{4} \succ x_{5} \approx x_{6} \succ x_{1} \approx x_{2} \approx x_{3} \\
& x_{4} \succ x_{5} \approx x_{6} \succ x_{1} \approx x_{2} \approx x_{3} \\
& x_{4} \succ x_{5} \approx x_{6} \succ x_{1} \approx x_{2} \approx x_{3} \\
& x_{4} \succ x_{5} \approx x_{6} \succ x_{1} \approx x_{2} \approx x_{3} \\
& x_{1} \approx x_{2} \approx x_{3} \approx x_{4} \approx x_{5} \approx x_{6}
\end{aligned}
$$

Using the similar method in case 3 , let $\alpha=\{0,0.1, \ldots, 0.9,1\}$, we can obtain the results as shown in Table 7 . Through this table, we can find that the results are different with different values of $\alpha$. But the results are highly consistent. When $\alpha=\{0.7,0.8,0.9,1\}$, the best selection is $x_{1}$ while the worst selections are $x_{2}$ and $x_{3}$. However, when $\alpha=$ $\{0,0.1, \ldots, 0.6\}$, the best selection is still $x_{4}$. In other words, if $0 \leq \alpha \leq 0.6$, the change of the value of $\alpha$ has no influence on our results. So using the similar way in case 3 to make decisions, we should take $0 \leq \alpha \leq 0.6$. 
Table 7. Ranking orders of alternative with different $\alpha$ in case 3 .

\begin{tabular}{cl}
\hline Different Value of $\alpha$ & Ranking Orders of Six Alternatives \\
\hline$\alpha=0$ & $x_{4} \succ x_{5} \approx x_{6} \succ x_{1} \approx x_{2} \approx x_{3}$ \\
$\alpha=0.1$ & $x_{4} \succ x_{5} \approx x_{6} \succ x_{1} \succ x_{2} \approx x_{3}$ \\
$\alpha=0.2$ & $x_{4} \succ x_{5} \approx x_{6} \succ x_{1} \succ x_{2} \approx x_{3}$ \\
$\alpha=0.3$ & $x_{4} \succ x_{1} \succ x_{5} \approx x_{6} \succ x_{2} \approx x_{3}$ \\
$\alpha=0.4$ & $x_{4} \succ x_{1} \succ x_{5} \approx x_{6} \succ x_{2} \approx x_{3}$ \\
$\alpha=0.5$ & $x_{4} \succ x_{1} \succ x_{2} \approx x_{3} \succ x_{5} \approx x_{6}$ \\
$\alpha=0.6$ & $x_{4} \succ x_{1} \succ x_{2} \approx x_{3} \succ x_{5} \approx x_{6}$ \\
$\alpha=0.7$ & $x_{1} \succ x_{4} \succ x_{5} \approx x_{6} \succ x_{2} \approx x_{3}$ \\
$\alpha=0.8$ & $x_{1} \succ x_{5} \approx x_{6} \succ x_{4} \succ x_{2} \approx x_{3}$ \\
$\alpha=0.9$ & $x_{1} \succ x_{5} \approx x_{6} \succ x_{4} \succ x_{2} \approx x_{3}$ \\
$\alpha=1$ & $x_{1} \succ x_{5} \approx x_{6} \approx x_{4} \approx x_{2} \approx x_{3}$ \\
\hline
\end{tabular}

In the Table 8, the sensitivity analysis of the IF rough sets model (case 1), $(I, T)$-IF rough sets model (case 2$)$ and $\left(R_{\widetilde{O}}, \widetilde{O}\right)$-IF rough sets model (case 3 ) are given.

Form the Table 8, we make some comparisons of the three models based on MCDM with IF information with different value of $\alpha$. Then we have the following results:

(1) The results of IF rough sets model, $(I, T)$-IF rough sets model and $\left(R_{\widetilde{O}^{\prime}} \widetilde{O}\right)$-IF rough sets model have the same choose that $x_{4}$ is the best alternative.

(2) We can find in case 1 and case 2, changing the value of $\alpha$ has no influence on our results (except $\alpha=0$ in case1, $\alpha=1$ in case 2). When $\alpha=0$, through comparison, IF rough sets model gives us is that six alternatives have the same weight, therefore, it is invalid in real life to making a decision. When $\alpha=1,(I, T)$-IF rough sets model gives us are that six alternatives have the same weight, therefore, it is invalid in real life to making a decision. Obviosly, $\left(R_{\widetilde{O}}, \widetilde{O}\right)$-IF rough sets model is better than IF rough sets model and $(I, T)$-IF rough sets model in this situation.

(3) When $\alpha=0.1$, the result of $\left(R_{\widetilde{O}}, \widetilde{O}\right)$-IF rough sets model and the result of $(I, T)$-IF rough sets model are highly consistent, When $\alpha=\{0.2,0.3\}$, the result of $\left(R_{\widetilde{O}^{\prime}} \widetilde{O}\right)$-IF rough sets model and the result of IF rough sets model are highly consistent. In other words, the result of $(I, T)$-IF rough sets model is one of many results of $\left(R_{\widetilde{O}}{ }^{\prime} \widetilde{O}\right)$-IF rough sets model.

Table 8. The comparison among ranking orders of alternative with different $\alpha$.

\begin{tabular}{|c|c|c|c|}
\hline Different Value of $\alpha$ & $\begin{array}{l}\text { IF Rough Sets Model } \\
\text { (Case 1) }\end{array}$ & $\begin{array}{c}(I, T) \text {-IF Rough Sets Model } \\
\text { (Case 2) }\end{array}$ & $\begin{array}{c}\left(R_{\widetilde{O}}, \widetilde{O}\right)-\text { IF Rough Sets } \\
\text { Model (Case 3) }\end{array}$ \\
\hline$\alpha=0$ & $x_{1} \approx x_{2} \approx x_{3} \approx x_{4} \approx x_{5} \approx x_{6}$ & $x_{4} \succ x_{5} \approx x_{6} \succ x_{1} \approx x_{2} \approx x_{3}$ & $x_{4} \succ x_{5} \approx x_{6} \succ x_{1} \approx x_{2} \approx x_{3}$ \\
\hline$\alpha=0.1$ & $x_{4} \succ x_{5} \succ x_{1} \approx x_{2} \approx x_{3} \approx x_{6}$ & $x_{4} \succ x_{5} \approx x_{6} \succ x_{1} \approx x_{2} \approx x_{3}$ & $x_{4} \succ x_{5} \approx x_{6} \succ x_{1} \succ x_{2} \approx x_{3}$ \\
\hline$\alpha=0.2$ & $x_{4} \succ x_{5} \succ x_{1} \approx x_{2} \approx x_{3} \approx x_{6}$ & $x_{4} \succ x_{5} \approx x_{6} \succ x_{1} \approx x_{2} \approx x_{3}$ & $x_{4} \succ x_{5} \approx x_{6} \succ x_{1} \succ x_{2} \approx x_{3}$ \\
\hline$\alpha=0.3$ & $x_{4} \succ x_{5} \succ x_{1} \approx x_{2} \approx x_{3} \approx x_{6}$ & $x_{4} \succ x_{5} \approx x_{6} \succ x_{1} \approx x_{2} \approx x_{3}$ & $x_{4} \succ x_{1} \succ x_{5} \approx x_{6} \succ x_{2} \approx x_{3}$ \\
\hline$\alpha=0.4$ & $x_{4} \succ x_{5} \succ x_{1} \approx x_{2} \approx x_{3} \approx x_{6}$ & $x_{4} \succ x_{5} \approx x_{6} \succ x_{1} \approx x_{2} \approx x_{3}$ & $x_{4} \succ x_{1} \succ x_{5} \approx x_{6} \succ x_{2} \approx x_{3}$ \\
\hline$\alpha=0.5$ & $x_{4} \succ x_{5} \succ x_{1} \approx x_{2} \approx x_{3} \approx x_{6}$ & $x_{4} \succ x_{5} \approx x_{6} \succ x_{1} \approx x_{2} \approx x_{3}$ & $x_{4} \succ x_{1} \succ x_{2} \approx x_{3} \succ x_{5} \approx x_{6}$ \\
\hline$\alpha=0.6$ & $x_{4} \succ x_{5} \succ x_{1} \approx x_{2} \approx x_{3} \approx x_{6}$ & $x_{4} \succ x_{5} \approx x_{6} \succ x_{1} \approx x_{2} \approx x_{3}$ & $x_{4} \succ x_{1} \succ x_{2} \approx x_{3} \succ x_{5} \approx x_{6}$ \\
\hline$\alpha=0.7$ & $x_{4} \succ x_{5} \succ x_{1} \approx x_{2} \approx x_{3} \approx x_{6}$ & $x_{4} \succ x_{5} \approx x_{6} \succ x_{1} \approx x_{2} \approx x_{3}$ & $x_{1} \succ x_{4} \succ x_{5} \approx x_{6} \succ x_{2} \approx x_{3}$ \\
\hline$\alpha=0.8$ & $x_{4} \succ x_{5} \succ x_{1} \approx x_{2} \approx x_{3} \approx x_{6}$ & $x_{4} \succ x_{5} \approx x_{6} \succ x_{1} \approx x_{2} \approx x_{3}$ & $x_{1} \succ x_{5} \approx x_{6} \succ x_{4} \succ x_{2} \approx x_{3}$ \\
\hline$\alpha=0.9$ & $x_{4} \succ x_{5} \succ x_{1} \approx x_{2} \approx x_{3} \approx x_{6}$ & $x_{4} \succ x_{5} \approx x_{6} \succ x_{1} \approx x_{2} \approx x_{3}$ & $x_{1} \succ x_{5} \approx x_{6} \succ x_{4} \succ x_{2} \approx x_{3}$ \\
\hline$\alpha=1$ & $x_{4} \succ x_{5} \succ x_{1} \approx x_{2} \approx x_{3} \approx x_{6}$ & $x_{1} \approx x_{2} \approx x_{3} \approx x_{4} \approx x_{5} \approx x_{6}$ & $x_{1} \succ x_{5} \approx x_{6} \approx x_{4} \approx x_{2} \approx x_{3}$ \\
\hline
\end{tabular}

The IF-rough model based on IF-overlap function presented in this paper is more flexible when dealing with specific application problems, and can reproduce the results obtained by other IF rough set models. According to the choice of different $\alpha$, different decision ordering can be obtained, so that the decision maker can have a better decision reference in practical problems. 


\section{Conclusions}

Inspired by the literature $[6,7,17,27]$, this paper puts forward the concept of IF-overlap function for the first time, and constructs an IF-rough set model based on IF-overlap function, which can be regarded as an extended form of IF-rough set based on IF triangular norm. On the one hand, the model retains the important properties of the original IF rough set model. On the other hand, the application range of IF rough sets is expanded and the flexibility is stronger. In order to solve MCDM problems in real life, the IF rough set model based on IF-overlap function is combined with IF TOPSIS method. The decision results show that the model has significant application value. Compared with other decision results, the model is more flexible, by changing the value of $\alpha(\alpha \in[0,1]$ is a level adjustment value), the new model can obtain the results of other models, which can reproduce most of the existing results and provide more reference for decision makers. As a further research topic, the variable precision intuitionistic fuzzy rough sets based on IF-overlap functions and the covering intuitionistic fuzzy rough sets [36-40] based on IF-overlap functions will be discussed in the following work, and applied to data mining and knowledge discovery, etc.

Author Contributions: The idea of this whole paper was put forward by X.Z., he also completed the preparatory work of the paper. X.W. analyzed the existing work of the intuitionistic fuzzy overlap function and rough sets and wrote the paper. T.L. finished proofreading. All authors have read and agreed to the published version of the manuscript.

Funding: This work was supported by National Science Foundation of China (Grant No. 61976130).

Institutional Review Board Statement: Not applicable.

Informed Consent Statement: Not applicable.

Data Availability Statement: Not applicable.

Conflicts of Interest: The authors declare no conflict of interest.

\section{References}

1. Zadeh, L.A. Fuzzy sets. Inf. Control. 1965, 8, 338-353. [CrossRef]

2. Meng, F.; Chen, S.M.; Tang, J. Group decision making based on acceptable multiplicative consistency of hesitant fuzzy preference relations. Inf. Sci. 2020, 524, 77-96. [CrossRef]

3. Liu, P.; Wang, P. Multilpe-attribute decision-making based on archimedean bonferroni operator of q-rung orthopair fuzzy numbers. IEEE Trans. Fuzzy Syst. 2018,27, 834-848. [CrossRef]

4. Zhang, K.Q.; Chen, Y.M.; Tang, C. Random fuzzy clustering granular hyperplane classifier. IEEE Access. 2020,12, 2169-3536.

5. Wang, J.Q.; Peng, J.J.; Zhang, H.Y. Outranking approach for multi-criteria decision-making problems with hesitant inter-valued fuzzy sets. Soft Comput. 2019, 23, 419-430. [CrossRef]

6. Wang, L.; Wang, H.; Xu, Z. The interval-valued hesitant pythagorean fuzzy sets and its application with extended topsis and choquet integral-based method. Int. J. Intell. Syst. 2019, 34, 1063-1085. [CrossRef]

7. Atanassov, K. Intuitionistic fuzzy sets. Fuzzy Sets Syst. 1986, 20, 87-96. [CrossRef]

8. Atanassov, K. On Intuitionistic Fuzzy Sets Theory; Springer: Berlin/Heidelberg, Germany, 2012.

9. Atanassov, K. Intuitionistic fuzzy sets. Int. J. Bioautom. 2016, 20, 1-6.

10. Pawlak, Z. Rough sets. Int. J. Comput. Inf. Sci. 1982, 11, 341-356. [CrossRef]

11. Dubois, D.; Prade, H. Rough fuzzy sets and fuzzy rough sets. Int. J. Gen. Syst. 1990, 17, 191-209. [CrossRef]

12. Morsi, N.N.; Yakout, M.M. Axiomatics for fuzzy rough sets. Fuzzy Sets Syst. 1998, 100, 327-342. [CrossRef]

13. Wang, C.Y.; Hu, B.M. Fuzzy rough sets based on generalized residuated lattices. Inf. Sci. 2013, 248, 31-49. [CrossRef]

14. Lin, Y.J.; Li, Y.W.; Wang, C.X. Attribute reduction for multi-label learning with fuzzy rough set. Knowl. Based Syst. $2018,152,51-61$. [CrossRef]

15. Wu, W.Z.; Yee, L.; Mi, J.S. On characterizations of (I,T)-fuzzy rough approximation operators. Fuzzy Sets Syst. 2005, 154, 76-102. [CrossRef]

16. Sun, B.Z.; Gong, Z.T.; Chen, D.G. Fuzzy rough set theory for the interval-valued fuzzy information systems. Inf. Sci. 2008, 178, 2794-2815. [CrossRef]

17. Atanassov, K. Intuitionistic Fuzzy Sets: Theory and Applications; Physica-Verlag: Heidelberg, Germany, 1999.

18. Vlachos, I.K.; Sergiadis, G.D. Intuitionistic fuzzy information-application to pattern recognition. Pattern Recognit. Lett. 2007, 28, 197-206. [CrossRef] 
19. Xu, Z.S. Some similarity measures of intuitionistic fuzzy sets and their applications to multiple attribute decision making. Fuzzy Optim. Decis. Mak. 2007, 6, 221-236. [CrossRef]

20. Traneva, V.; Tranev, S. Multidimensional intuitionistic fuzzy InterCriteria analysis in the restaurant. J. Intell. Fuzzy Syst. 2020. [CrossRef]

21. Traneva, V.; Tranev, S. Intuitionistic Fuzzy Two-factor Variance Analysis of Movie Ticket Sales. J. Intell. Fuzzy Syst. 2021. [CrossRef]

22. Bustince, H.; Burillo, P. Structures on intuitionistic fuzzy relations. Fuzzy Sets Syst. 1996, 78, 293-303. [CrossRef]

23. Deschrijver, G.; Cornelis, C.; Kerre, E. On the representation of intuitionistic fuzzy t-norm and t-conorms. IEEE Trans. Fuzzy Syst. 2004, 12, 45-61. [CrossRef]

24. Zhou, L.; Wu, W.Z. On generalized intuitionistic fuzzy rough approximation operators. Inf. Sci. 2008, 178, 2448-2465. [CrossRef]

25. Decock, M. A Thorough Study of Linguistuc Modifiers in Fuzzy Set Theory. Ph.D. Thesis, Ghent University, Ghent, Belgium, 2002.

26. Cornelis, C.; Cock, M.; Kerre, M.E. Intuitionistic fuzzy rough sets: At the crossroads of imperfect knowledge. Expert Syst. Int. J. Knowl. Eng. Neural Netw. 2003, 20, 260-269. [CrossRef]

27. Zhang, X.H.; Zhou, B.; Li, P. A general frame for intuitionistic fuzzy rough sets. Inf. Sci. 2012, 216, 34-39. [CrossRef]

28. Bustince, H.; Fernandez, J.; Mesiar, R. Overlap Index, Overlap Functions and Migrativity. In Proceedings of the Joint 2009 International Fuzzy Systems Association Word Congress and 2009 European Society of Fuzzy Logic and Technology Conference, Lisbon, Portugal, 20-24 July 2009; Volume 65, pp. 300-305.

29. Bustince, H.; Barrenecha, E.; Pagola, M. Image thresholding using restrited equivalent functions and maximizing the measures of similarity. Fuzzy Sets Syst. 2007, 158, 496-516. [CrossRef]

30. Amo, A.; Montero, J.; Biging, G. Fuzzy classification systems. Eur. J. Oper. Res. 2004, 156, 495-507. [CrossRef]

31. Elkano, M.; Galar, M.; Sanz, J. Enhancing multiclass classification in FARC-HD fuzzy classifier: On the synergy between n-dimensional overlap functions and decomposition strategies. IEEE Trans. Fuzzy Syst. 2015, 23, 1562-1580. [CrossRef]

32. Qiao, J.; Hu, B.Q. On interval additive generators of interval overlap functions and interval grouping functions. Fuzzy Sets Syst. 2017, 323, 19-55. [CrossRef]

33. Bedregal, B.; Bustince, H.; Palmeira, E. Generalized interval-valued OWA operators with interval weights derived from interval.Cvalued overlap functions. Int. J. Approx. Reason. 2017, 90, 1-16. [CrossRef]

34. Tiago, D.C.; Graliz, P.D. General interval-valued overlap functions and interval-valued overlap indices. Inf. Sci. 2020, 527, 27-50.

35. Pei, Z.; Zheng, L. A novel approach to multi-attribute decision making based on intuitionistic fuzzy sets. Expert Systems with Applications, 2012, 39, 2560-2566. [CrossRef]

36. Zhang, L.; Zhan, J.M.; Xu, Z.S. Covering-based generalized IF rough sets with applications to multi-attribuate decision-making. Inf. Sci. 2019, 478, 275-302. [CrossRef]

37. Zhan, J.M.; Sun, B.; Alcantud, J.R. Covering based multigranulation $(\varphi, \tau)$-fuzzy rough set models and applications in multattribute group decisionmaking. Inf. Sci. 2019, 476, 290-318. [CrossRef]

38. Yao, Y.Y.; Yao, B. Covering based rough sets approximations. Inf. Sci. 2012, 200, 91-107. [CrossRef]

39. Zhang, X.H.; Wang, J.Q. Fuzzy $\beta$-covering approximation space. Int. J. Approx. Reason. 2020, 26, 27-47. [CrossRef]

40. Zhang, X.H.; Wang, J.Q.; Zhan, J.M.; Dai, J.H. Fuzzy measures and Choquet integrals based on fuzzy covering rough sets. IEEE Trans. Fuzzy Syst. 2021. [CrossRef] 